\section{Modelling Yagi-Uda antennas using point source approximation}

\section{H. Ebersbach, D.V. Thiel and M. Leckenby}

\begin{abstract}
A point source approximation is shown to be an efficient algorithm for calculating the near and far fields of Yagi-Uda antennas for the evaluation of the human radiation exposure zone boundaries. Results of a 12-element Yagi-Uda antenna compared to numerical electromagnetics code show that the modelled antenna field strength and front-to-back ratio were calculated to within 0.10 and $0.34 \mathrm{~dB}$, respectively, while the occupational and general public exposure zone boundary distances were calculated to within 0.23 and $0.14 \mathrm{~m}$, respectively.
\end{abstract}

Introduction: An efficient calculation method for the near and far fields of dipole and multi-element Yagi-Uda antennas is required for the evaluation of radiation exposure zone boundary distances. Traditional tools for calculating the fields of wire antennas include the method of moments such as the numerical electromagnetics code (NEC) [1], and other modelling packages based on the finite difference time domain and finite element method algorithms. While these tools are accurate for most applications, a need exists for a more rapid calculation of the fields surrounding wireless communication sites with large numbers of antenna structures. The strategy of the algorithm presented in this Letter is to calculate the electric field strength in three-dimensional space from a single radiating point source located at the centre of each element of the Yagi-Uda antenna. The fields surrounding the antenna are then calculated from these Huygen's wavelets, and the exposure zone boundaries subsequently evaluated from the field strength contour.

Theory: Conventional analytical expressions for calculating the field strength of a dipole excited by a sinusoidal current distribution have been developed by Schelkunoff [2] and included in many subsequent antenna and electromagnetic textbooks. The field equations for $E_{Z}, H_{\varphi}$ and $E_{\rho}$ show spherical waves originating from three points in each expression: the central feed point of the dipole and the two end points. While the two end points have a field related to the current, it is well known that the current magnitude at these two points approaches zero. The electric field strength calculation using [2] would therefore require the summation of fields from six radiating point sources for a dipole antenna. The radiation from a parasitic element has not been calculated in this way, although some possibilities have been explored previously [3]. In this algorithm, a single radiating point source is placed at the centre of each Yagi-Uda antenna element. The intensity of each source is the current in each element calculated from mutual coupling equations.

The numerical technique of Baker and LaGrone [4] is used to calculate the mutual impedances between all Yagi-Uda antenna array elements. This mutual EMF-method algorithm calculates the mutual impedance between thin dipoles of any length and geometrical configuration. The self impedance of elements with finite radii is the mutual impedance of two identical wire antenna elements with an apparent inter-element-spacing equal to the element radius.

From these calculated mutual and self impedances, the input impedance of the Yagi-Uda antenna is determined. The specified transmitter input power allows computation of the input voltage of the active element. By re-solving the network equations, the complex current amplitudes of the active and parasitic Yagi-Uda antenna elements are determined.

Radiating point sources located at the centre of each Yagi-Uda antenna element with the source strengths equal to the calculated complex current amplitudes allows the computation of the field strength of the antenna by superposition of the field strength contributions of each radiating point source.

Results: The algorithm was implemented in $\mathrm{C}++$ and tested on various dipole and Yagi-Uda antenna arrays. Amongst the antennas tested, was a 12-element Yagi-Uda antenna, see Fig. 1, chosen from the literature [5]. Assuming sinusoidal current distributions, the mutual and self impedances of the individual antenna elements were calculated using the Baker and LaGrone algorithm. The input impedance of the antenna was $34.27+j 50.41 \Omega$. Assuming a transmitting input power to the antenna as $50 \mathrm{~W}$, the excitation voltage of the antenna feed-element was evaluated as $48.79+j 25.83 \mathrm{~V}$. Finally the complex current amplitudes of the active and each parasitic antenna array element were calculated.

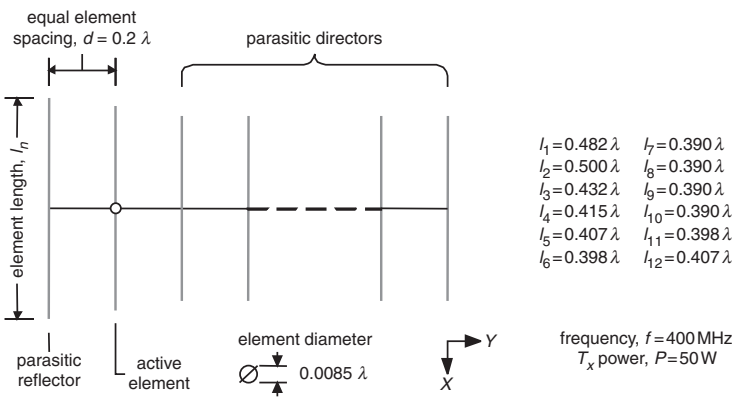

Fig. 1 12-element Yagi-Uda antenna

Figs. 2 and 3 show plots of the electric field magnitude, calculated at a distance of $25 \mathrm{~m}$ from the antenna feed point in the $E$ - and $H$-planes, respectively. These far-field results are compared to those obtained using NEC [1].

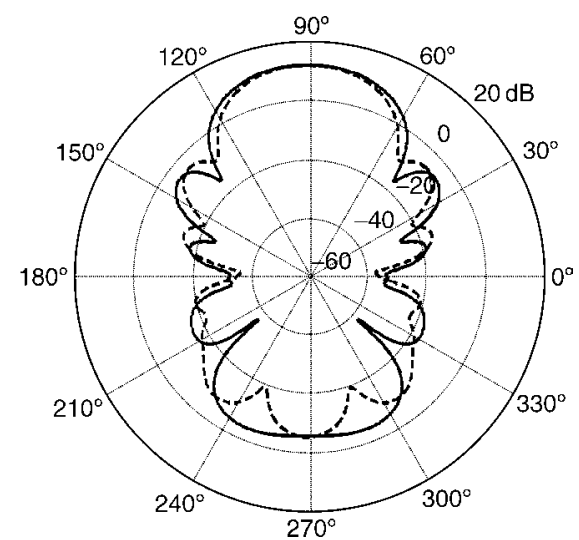

Fig. 2 E-plane far-field radiation plot in $d B$ calculated using point source model and NEC

point source model NEC

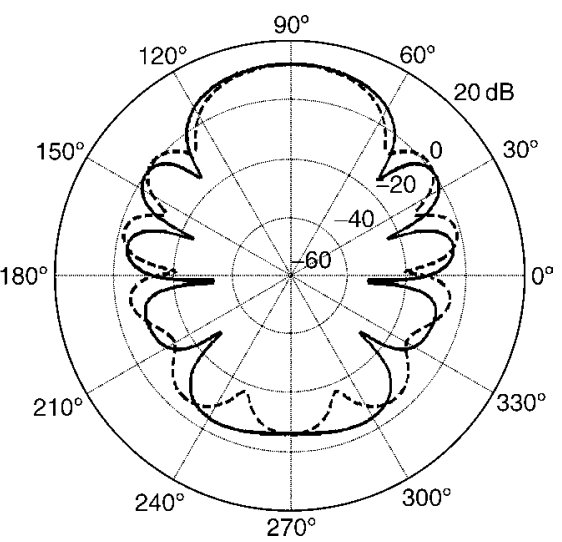

Fig. 3 H-plane far-field radiation plot in $d B$ calculated using point source model and NEC

- point source model NEC

The antenna field strength and front-to-back ratio were calculated to be 13.80 and $19.64 \mathrm{~dB}$, respectively, and are within 0.10 and $0.34 \mathrm{~dB}$ of the respective NEC results. While some minor discrepancies between the results of the presented algorithm and the equivalent NEC calculations are observed in these far-field plots, these lie within the sidelobe regions of the radiation patterns and are therefore of lesser significance to the radiation exposure zone boundary calculations. 
To calculate the occupational and non-occupational radiation exposure zone boundary distances, the electric field strength was modelled within an area $10 \times 10 \mathrm{~m}$ surrounding the antenna. At the frequency of $400 \mathrm{MHz}$, the reference levels for time averaged exposure to RMS electric fields for the occupational and non-occupational categories according to the ARPANSA [6] standard are 61.4 and $27.4 \mathrm{~V} / \mathrm{m}$, respectively. These field strength contours are shown in Fig. 4 alongside equivalent NEC based calculations.

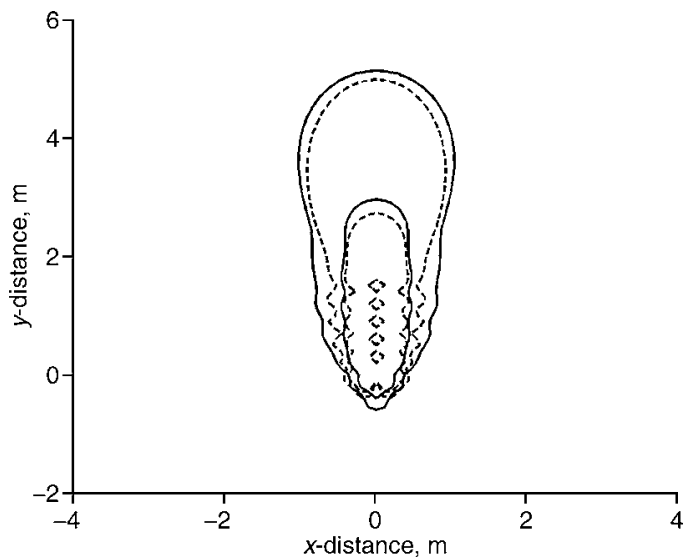

Fig. 4 Occupational (inner lines) and non-occupational (outer lines) E-field exposure zone boundaries calculated using point source model and $N E C$

point source model

--- NEC

In Fig. 4 the exposure zone boundary distances at the antenna boresight are 2.95 and $5.11 \mathrm{~m}$ for the occupational and non-occupational boundaries, respectively. These distances are within 0.23 and $0.14 \mathrm{~m}$ of their respective NEC calculated counterparts.
Conclusions: An efficient algorithm for calculating the near and far fields of Yagi-Uda antennas for the evaluation of the human radiation exposure zone boundary distances has been shown. The algorithm has been demonstrated with a 12-element Yagi-Uda antenna. While some minor discrepancies within the sidelobe regions of the far-field radiation plots compared to NEC are evident, the agreement of the exposure zone boundary distances is good. The algorithm has been tested on a range of other antennas where similar accuracy has been achieved.

(C) IEE 2004

4 June 2004

Electronics Letters online no: 20045690

doi: 10.1049/el:20045690

H. Ebersbach and D.V. Thiel (Radio Science Laboratory, Griffith University, Nathan, Queensland 4111, Australia)

E-mail: h.ebersbach@griffith.edu.au

M. Leckenby (iCOMMS Pty Ltd, Mitchelton, Queensland 4053, Australia)

\section{References}

1 Lawrence Livermore National Laboratory: 'Numerical electromagnetics code (NEC)', Livermore, CA, USA

2 Schelkunoff, S.A.: 'Electromagnetic waves' (D. van Nostrand Company Inc., New York, NY, USA, 1943), pp. 369-371

3 Ebersbach, H., and Thiel, D.V.: 'Yagi antenna modelling using a number of radiating point sources'. 8th Australian Symp. on Antennas, CSIRO, Sydney, Australia, February 2003, p. 36

4 Baker, H.C., and LaGrone, A.H.: 'Digital computation of the mutual impedance between thin dipoles', IRE Trans. Antennas Propag., 1962, 10, (3), pp. 172-178

5 Viezbicke, P.P.: 'Yagi antenna design' (National Bureau of Standards Technical Note 688, 1976)

6 Australian Radiation Protection and Nuclear Safety Agency (ARPANSA): 'Radiation protection standard: maximum levels to radiofrequency fields $-3 \mathrm{kHz}$ to $300 \mathrm{GHz}$ ' (ARPANSA, May 2002, Yullambie, Australia) 